\title{
Aspectos químicos e biológicos do gênero Lippia enfatizando Lippia gracilis Schauer
}

\author{
S. V. F. Gomes, P. C. L. Nogueira, V. R. S. Moraes* \\ LABORGANICS, Departamento de Química, Universidade Federal de Sergipe, Av. Marechal \\ Rondon, s/n, 49100-000, São Cristovão-SE, Brasil. \\ *valrsmoraes@uol.com.br
}

RESUMO

\begin{abstract}
“Aspectos químicos e biológicos do gênero Lippia enfatizando Lippia gracilis Schauer”. Esta revisão apresenta um estudo sobre os principais constituintes químicos e aspectos biológicos de espécies do gênero Lippia enfatizando a Lippia gracilis Schauer. O gênero Lippia (Verbenaceae) possui aproximadamente 200 espécies de ervas, arbustos e pequenas árvores, cujos maiores centros de dispersão se encontram em países das Américas do Sul e Central, como também em territórios da África tropical. Inúmeras espécies de Lippia são usadas na medicina popular para o tratamento de resfriados, gripes, bronquites e tosse. As pesquisas referentes à composição química das espécies de Lippia evidenciam, principalmente, os constituintes voláteis. Entretanto, outras substâncias como alcalóides, taninos, flavonóides, iridóides e naftoquinonas também são citados. Sobre a espécie Lippia gracilis Schauer, existem vários estudos dos constituintes voláteis, apresentando como principais compostos o timol e carvacrol, que tem forte atividade antimicrobiana contra fungos e bactérias. No entanto, as pesquisas sobre os constituintes fixos são poucos, evidenciando a grande necessidade de trabalhos que enfoquem outras classes de compostos.
\end{abstract}

Palavras-chave: Lippia gracilis; óleo essencial; flavonóides; iridóides; naftoquinonas

\section{Introdução}


O uso de plantas com fins terapêuticos é uma tradição milenar presente nas culturas de várias nações, constituindo, ainda hoje, um recurso alternativo de grande aceitação [1].

Entre as plantas utilizadas como medicinais, destacam-se as espécies da Família Verbenaceae pertencente à ordem Lamiales. Esta Família compreende 35 gêneros e 1.035 espécies, muitos exclusivamente brasileiros, com distribuição tropical e subtropical. Os gêneros mais representativos em número de espécies são: Verbena, Lippia, Citharexylum, Stachytarpheta, Glandularia e Duranta [2].

O gênero Lippia, o segundo maior da família Verbenaceae, possui aproximadamente 200 espécies de ervas, arbustos e pequenas árvores, cujos maiores centros de dispersão se encontram em países das Américas do Sul e Central, como também em territórios da África tropical [3-4].

Os principais centros de diversidade específica do gênero Lippia estão localizados no México e Brasil, onde o maior número de espécies se encontra na Cadeia do Espinhaço, localizada nos estados de Minas Gerais, Bahia e Goiás [5].

Aproximadamente 120 espécies de Lippia encontram-se no Brasil, distribuídas no cerrado e caatinga, onde se destacam por seu aspecto chamativo no período da floração e por seu aroma forte e geralmente agradável. Destas espécies, a L. alba (Mill.) N. E. Brown (erva cidreira ou falsa melissa) é a mais conhecida e utilizada devido as suas propriedades medicinais [6].

No nordeste do país as espécies de Lippia são usadas na medicina popular para o tratamento de resfriados, gripes, bronquites e tosse. Em muitos casos, as partes usadas são as folhas e flores na forma de infusão ou decocto administradas oralmente ou através de emplastos [4, 6].

Inúmeras espécies de Lippia são empregadas na medicina tradicional no tratamento de patologias diversas e, como resultado, muitas têm sido investigadas do ponto de vista farmacológico revelando importantes propriedades tais como, ação sedativa, antiespamódica, estomáquica, antiinflamatória e antipirética de L. alba (Mill.) N. E. Brown [7]; efeito antisséptico, antiinflamatório e cicatrizante do extrato aquoso de L. sidoides Cham [8]; ação contra a malária, no tratamento de hipertensão e combate à sarna de L. multiflora Moldenke [9-10] e tratamento da tosse e bronquite de L. dulcis Trevir [11].

Além de suas propriedades medicinais, as folhas da maioria das espécies são utilizadas na preparação de alimentos [12]. Neste aspecto é interessante ressaltar a importância de L. dulcis Trevir., cujo principal componente das folhas e flores é a (+)-hernandulcina (1), uma molécula 1.000 vezes mais doce que a sacarose $[4,11,13]$. 
Algumas espécies de Lippia têm sido utilizadas também no reflorestamento de áreas mineradas. Lippia origanoides H.B.K., por exemplo, tem sido usada como espécie pioneira em regiões de minério de ferro que foram desativadas ou abandonadas na Venezuela [14].

Segundo Pascual e colaboradores [4], algumas espécies de Lippia, ao longo dos anos, tem apresentado problemas de nomenclatura botânica devido à dificuldade em torno da correta identificação de certas espécies causando a utilização de diversas sinonímias para uma mesma espécie em artigos científicos. L. alba (Mill.) N. E. Br., por exemplo, apresenta várias sinonímias e pode receber o nome de L. geminata microphylla Griseb, L. germinata H.B.K., L. globiflora Kuntze, L. lantanoides Coult, Lantana alba Mill e Phyla germinata H.B.K..

Com o propósito de contribuir para uma solução, a sistematização das informações referentes à denominação botânica de espécies do gênero Lippia foi realizada pela revisão do registro dessas espécies no INPI (The International Plant Names Index). Nesta revisão algumas espécies vêm classificadas, ora como binômio válido, ora como sinonímias. Dentre estas espécies, a L. gracilis apresentou dois binômios válidos, sendo eles: L. gracilis Schauer e L. gracilis Phil.

\section{A espécie Lippia gracilis Schauer}

Lippia gracilis Schauer é uma planta aromática endêmica do nordeste brasileiro, próprio da vegetação semi-árido, conhecida popularmente como alecrim-da-chapada ou alecrim-de-serrote. É encontrada predominantemente nos estados da Bahia, Sergipe e Piauí [15]. Apresenta-se como um arbusto de aproximadamente $2,5 \mathrm{~cm}$ de altura, bem ramificada, de folhas pequenas e flores brancas, ambas bastante odoríferas.

As folhas de L. gracilis são ricas em óleo essencial com significativa atividade antimicrobiana contra fungos e bactérias [16]. Além disso, elas são bastante usadas nas infecções da garganta e boca, problemas vaginais, tratamento da acne, panos brancos, impigens, caspa, queimaduras e feridas [17-18].

\section{Constituintes químicos}

Os estudos referentes à composição química das espécies de Lippia evidenciam, principalmente, os constituintes voláteis. Entretanto, outras substâncias como flavonóides, iridóides e naftoquinonas também são citados com frequiência [19-25].

Alguns alcalóides foram identificados na $L$. dulcis Trevir, L. germinata H.B.K., L. nodiflora (L.) Michx e L. turbinata Griseb [26-28] enquanto que taninos são encontrados na L. alba (Mill.) N.E. Brown [28-29]. 
Triterpenos e derivados de esteróides, geralmente identificados como saponinas, foram relatados na L. alba (Mill.) N.E. Brown, L. javanica (N.L.Burm.) Spreng., L. rehmani H.H.W. Pearson e L. turbinata Griseb [26, 28-31].

Atualmente existe um grande número de estudos fitoquímicos e farmacológicos de L. alba (Mill.) N.E. Brown e L. multiflora Moldenke, no entanto para as outras espécies os estudos são poucos no que se refere aos aspectos químicos.

\section{Óleo essencial}

A composição química do óleo essencial de muitas espécies de Lippia tem sido investigada através de Cromatografia Gasosa e, com base nos dados publicados, percebe-se que limoneno (2), $\beta$-cariofileno (3), p-cimeno (4), cânfora (5), linalool (6), $\alpha$-pineno (7) e timol (8) são os componentes que aparecem com maior freqüência [4, 32].

Além destes, foi isolado de L. dulcis a (+)-hernandulcina (1), (+)-4ß-hidroxihernandulcina e (-)epihernandulcina (9) [11-12]. Outro estudo da mesma planta relatou a presença de seis novos sesquiterpenos do tipo bisabolano das partes áreas, o peroxilippidulcinas A-C (10-12), peroxilippidulcinas B (13), epilippidulcinas B (14) e C (15), lippidulcina A (16) e epilippidulcina A (17) [33].

No gênero Lippia, a secreção de óleos essenciais tem sido associada à presença de tricomas, os quais normalmente são de formas variadas entre grupos vegetais, mas em geral uniformes dentro de um mesmo táxon [34].

Os constituintes dos óleos essenciais são muito instáveis a presença de luz, calor e umidade, conseqüentemente o horário de colheita do material vegetal pode influenciar direta ou indiretamente nos processos do metabolismo secundário que resultam em variações quantitativas e qualitativas do óleo essencial [34].

A composição química do óleo de L. gracilis Schauer mostra flutuações quantitativas dos componentes majoritários, provavelmente devido a condições genéticas, em função do local e condições em que a planta é cultivada.

Neves e colaboradores [35] realizaram por Cromatografia Gasosa (CG) e Cromatografia Gasosa acoplada a Espectrometria de Massas (CG/EM) a determinação dos compostos químicos presentes no óleo essencial de L. gracilis Schauer em dois locais da caatinga de Pernambuco, Buíque e Ouricuri. O estudo mostrou a presença de carvacrol (18) e p-cimeno (4) como 
constituintes principais em Buíque e timol (8), $\gamma$-terpineno (19) e 4-metoxi-acetofenona (20) como compostos majoritários em Ouricuri.

O óleo essencial de L. gracilis também tem sido investigado em outros estados do nordeste do Brasil, como Ceará, Piauí e Sergipe. No Ceará o óleo essencial foi caracterizado por timol (8) (30,6\%) e p-cimeno (4) (10,7\%) como componentes majoritários [16], no Piauí tem-se o carvacrol (18) (47,7\%) e o $p$-cimeno (4) (19,2\%) [18] e em Sergipe, o carvacrol (18) (23,52\%), p-cimeno (4) (15,82\%), $\gamma$-terpineno (19) $(14,17 \%)$ e mentol (21) $(10,97 \%)$ [36].

Os compostos químicos presentes no óleo essencial, principalmente os monoterpenos carvacrol e timol, das espécies do gênero Lippia tem apresentado forte ação contra bactérias e fungos. Neste contexto, a L. origanoides H.B.K. apresentou atividade frente a bactérias como Staphylococcus aureus meticilino resistente e L. sidoides Cham. contra Escherichia coli, Enterococcus faecalis, Salmonela enteritidis, Serratia marcescens, Candida albicans e Mycobacterium smegmatis [12, 16-17, 37-38].

Um estudo realizado por Albuquerque e colaboradores [39] mostrou que o óleo essencial da $L$. gracilis Schauer possui atividade antimicrobiana contra fungos e bactérias endofíticas de helicônias. O óleo apresentou uma inibição de $100 \%$ contra os fungos Geotrichum candidum, Trichoderma viride, Torula herbarum, Paecillomyces sp, Aspergillus nidulans, Fusicoccum sp, Aspergillus flavus e Paecillomyces aeruginens; 95,58\% contra Curvularia lunata e de 89,40\% contra Aspergillus niger.

As bactérias Salmonela choleraceuis-diarizonae, Enterobacter asburiae, Bacillus thuringiensis, Bacillus pumilis, Kleibsiella pneumoniae, Enterobacter hormaechei e Bacillus cereus foram todas inibidas na presença do óleo essencial de L. gracilis. Essa ação foi associada à presença de dois monoterpenos fenólicos, o carvacrol $(41,77 \%)$ e o timol $(10,13 \%)$.

\section{Fitoconstituintes}

\section{Flavonóides}

Os flavonóides representam um dos grupos fenólicos mais importantes e diversificados entre os produtos de origem vegetal, aparecendo com relativa freqüência em espécies deste gênero. Eles possuem a capacidade de modular a atividade de enzimas e afetar o comportamento de muitos sistemas celulares, sugerindo que muitas espécies podem possuir efeitos anti-hepatotóxico, antialérgico, antiinflamatório, antiosteoporótipo e até antitumoral [40]. 
A maioria dos flavonóides identificados no gênero Lippia são flavonas, sendo as mais freqüentes as 6-hidroxiflavonas, metoxiflavonas e alguns sulfatos de flavonas (mono e disulfatos) [4].

Pascual e colaboradores [4] relataram a presença de duas flavanonas, a naringenina (22) e a pinocembrina (23), na espécie L. graveolens. Em 2007, Lin e colaboradores [25] identificaram nove novas flavanonas do extrato hidroalcoólico $\left(\mathrm{MeOH}: \mathrm{H}_{2} \mathrm{O}-70: 30 \mathrm{v} / \mathrm{v}\right)$ das folhas de $L$. graveolens por LC-DAD-ESI/MSD.

O estudo a respeito dos flavonóides foi realizado principalmente nas espécies L. citriodora (Ort.) H.B.K. e L. nodiflora (L.) Michx. No total, são aproximadamente 56 ocorrências de flavonóides no gênero Lippia, sendo 45 derivados de flavonas (24) e 11 derivados de flavanonas (25), como mostrado na tabela 1.

TABELA 1: Flavonóides isolados de espécies do gênero Lippia.

\begin{tabular}{llc}
\hline \multicolumn{1}{c}{ Compostos } & \multicolumn{1}{c}{ Espécies } & Autor(es)/Ano \\
\hline $\begin{array}{l}\text { apigenina } \\
\text { 5,7,4'-OH flavona) }\end{array}$ & $\begin{array}{l}\text { L. citriodora } \\
\text { L. graveolens }\end{array}$ & {$[19,21-22,25]$} \\
\hline apigenina-7-O-glicosil & L. graveolens & {$[25]$} \\
\hline apigenina-7-glucoronil-glicosil & L. triphylla & {$[55-56]$} \\
\hline apigenina-6-OMe-7-metil éter & L. sidoides & {$[57]$} \\
\hline $\begin{array}{l}\text { cirsiliol } \\
\text { (5,3',4'-OH-6,7-OMe flavona) }\end{array}$ & L. citriodora & {$[19,21-22]$} \\
\hline $\begin{array}{l}\text { cirsimaritina } \\
\text { (5,4'-OH-6,7-OMe flavona) }\end{array}$ & $\begin{array}{l}\text { L. citriodora } \\
\text { L. sidoides }\end{array}$ & {$[19,21-22,33,42,57]$} \\
\hline $\begin{array}{l}\text { crisoeriol } \\
\text { (5,7,4'-OH-3-OMe flavona) }\end{array}$ & L. dulcis & \\
\hline diosmetina & Litriodora & {$[19,21-22,25]$} \\
(5,7,3'-OH-4'-OMe flavona) & L. citriodora & {$[19,21-22,25]$} \\
\hline diosmetina-7-glucoronil-glicosil & L. triphylla & {$[55,56]$} \\
\hline $\begin{array}{l}\text { eupafolina } \\
\text { (5,7,3',4'-OH-6-OMe flavona) }\end{array}$ & L. citriodora & {$[19,21]$} \\
\hline $\begin{array}{l}\text { eupatorina } \\
\text { (5,3'-OH-6,7,4'-OMe flavona) }\end{array}$ & $\begin{array}{l}\text { L. citriodora } \\
\text { L. dulcis }\end{array}$ & {$[19,21-22,25,33]$} \\
\hline $\begin{array}{l}\text { eupatilina } \\
\text { 5,7-OH-6,3',4'-OMe flavona) }\end{array}$ & L. dulcis & {$[33]$} \\
\hline $\begin{array}{l}\text { galangina } \\
\text { (3,5,7-OH flavona) }\end{array}$ & L. graveolens & {$[25]$} \\
\hline metil-galangina & L. graveolens & {$[25]$} \\
\hline jaceosidina & L. nodiflora & {$[56]$} \\
\hline (luteolina-6-OMe-3'-metil éter) & L. nodiflora & \\
jaceosidina-7-sulfato & & \\
\hline
\end{tabular}




\begin{tabular}{|c|c|c|}
\hline jaceosidina-7,4'-disulfato & L. nodiflora & {$[56]$} \\
\hline $\begin{array}{l}\text { luteolina } \\
\left(5,7,3^{\prime}, 4^{\prime}-\mathrm{OH} \text { flavona }\right)\end{array}$ & $\begin{array}{l}\text { L. citriodora } \\
\text { L. graveolens } \\
\text { L. sidoides } \\
\end{array}$ & {$[19,21-22,25,42,57,58]$} \\
\hline luteolina 6-OH & $\begin{array}{l}\text { L. citriodora } \\
\text { L. graveolens } \\
\text { L. nodiflora } \\
\end{array}$ & {$[19,21-22,55]$} \\
\hline luteolina-7-O- $\beta$-glicosil & $\begin{array}{l}\text { L. citriodora } \\
\text { L. graveolens }\end{array}$ & {$[19,21-22,25]$} \\
\hline luteolina-6-OH-6-sulfato & L. citriodora & {$[19,21-22]$} \\
\hline luteolina-6-OH-7-sulfato & L. citriodora & {$[19,21-22]$} \\
\hline luteolina-6-OH-6,7-disulfato & L. citriodora & {$[19,21-22]$} \\
\hline luteolina-6-OH-7-O-hexosil & L. graveolens & {$[25]$} \\
\hline luteolina-6-OH-7-O-ramminosil & L. graveolens & [25] \\
\hline luteolina-glucoronil-glicosil & L. triphylla & [55-56] \\
\hline $\begin{array}{l}\text { lippiflorina A } \\
\text { (luteolina-6-OH-7-arabinosil) }\end{array}$ & L. citriodora & {$[19,21-22]$} \\
\hline $\begin{array}{l}\text { lippiflorina B } \\
\text { (luteolina-6-OH-7-arabinosil-4'- } \\
\text { ramnosil) }\end{array}$ & L. citriodora & {$[19,21-22]$} \\
\hline luteolina-7-glicosil & L. citriodora & {$[19,21-22]$} \\
\hline luteolina-6-OH-7-apiosídeo & L. citriodora & {$[19,21-22]$} \\
\hline $\begin{array}{l}\text { nepetina } \\
\text { (luteolina-6-OMe) }\end{array}$ & L. nodiflora & {$[56]$} \\
\hline nepetina-3',4'-disulfato & L. nodiflora & [56] \\
\hline nepetina-7-sulfato & L. nodiflora & [56] \\
\hline $\begin{array}{l}\text { nodifloretina } \\
\text { (luteolina-6-OH-3'-metil éter) }\end{array}$ & L. nodiflora & {$[56]$} \\
\hline nodifloretina-7-sulfato & L. nodiflora & {$[56]$} \\
\hline nodifloretina-6,7-disulfato & L. nodiflora & [56] \\
\hline $\begin{array}{l}\text { pectolinarigenina } \\
\left(5,7-\mathrm{OH}-6,4^{\prime}-\mathrm{OMe} \text { flavona) }\right.\end{array}$ & $\begin{array}{l}\text { L. citriodora } \\
\text { L. dulcis }\end{array}$ & {$[19,21-22,33]$} \\
\hline $\begin{array}{l}\text { salvigenina } \\
\text { (5-OH-6,7,4'-OMe flavona) }\end{array}$ & $\begin{array}{l}\text { L. citriodora } \\
\text { L. dulcis }\end{array}$ & {$[19,21-22,33]$} \\
\hline $\begin{array}{l}\text { scutellarein } \\
(5,6,7,4 \text { '-OH flavona })\end{array}$ & L. graveolens & {$[25]$} \\
\hline scutellarein-7-O-hexosil & L. graveolens & {$[25]$} \\
\hline scutellarein-6-metil & L. graveolens & [25] \\
\hline scutellarein-6,7-dimetil & L. graveolens & {$[25]$} \\
\hline 5-4-OH-6,7- OMe flavona & L. sidoides & {$[22,42,57]$} \\
\hline 5,3'-OH-6,7,4',5'-OMe flavona & L. dulcis & {$[33]$} \\
\hline $\begin{array}{l}\text { quercetina } \\
\left(3,5,7,3^{\prime}, 4^{\prime}-\text { OH flavona }\right)\end{array}$ & $\begin{array}{l}\text { L.aff. gracilis } \\
\text { L. dulcis } \\
\text { L. graveolens }\end{array}$ & {$[19,21-23,25,33,42,57-58]$} \\
\hline
\end{tabular}




\begin{tabular}{|c|c|c|}
\hline & L. sidoides & \\
\hline $\begin{array}{l}\text { eriodictiol } \\
\left(5,7,3^{\prime}, 4^{\prime}-\mathrm{OH} \text { flavanona }\right)\end{array}$ & L. graveolens & [25] \\
\hline eriodictiol-7-O-glicosil & L. graveolens & [25] \\
\hline $\begin{array}{l}\text { naringenina } \\
(5,7,4 \text { '-OH flavanona) }\end{array}$ & L. graveolens & {$[20,25]$} \\
\hline $\begin{array}{l}\text { pinocembrina } \\
(5,7-\mathrm{OH} \text { flavanona) }\end{array}$ & L. graveolens & {$[20,25]$} \\
\hline $\begin{array}{l}\text { sakuranetina } \\
\left(5,4^{\prime}-\mathrm{OH}-7-O M e \text { flavanona) }\right.\end{array}$ & L. graveolens & {$[25]$} \\
\hline $5,6,7,3^{\prime}, 4^{\prime}-\mathrm{OH}$ flavanona & L. graveolens & [25] \\
\hline 5,6,7,3',4'-OH-7-O-hexosil flavanona & L. graveolens & [25] \\
\hline $3,5,6,7,4^{\prime}-\mathrm{OH}$ flavanona & L. graveolens & [25] \\
\hline 3,5,6,7,4'-OH-7-O-hexosil flavanona & L. graveolens & {$[25]$} \\
\hline 3,5,6,7,4'-OH-2-O-hexosil flavanona & L. graveolens & [25] \\
\hline $\begin{array}{l}\text { taxifolina } \\
\left(3,5,7,3^{\prime}, 4^{\prime}-\mathrm{OH} \text { flavanona) }\right.\end{array}$ & $\begin{array}{l}\text { L. graveolens } \\
\text { L. sidoides }\end{array}$ & {$[25,57-58]$} \\
\hline
\end{tabular}

\section{Naftoquinonas}

As naftoquinonas não são freqüentes nas espécies do gênero Lippia. No entanto, no extrato metanólico das folhas e ramos de L. sidoides foi isolado o lapachenol (26), o isocatalponol e o 6oxo-3,4,4a,5-tetrahidro-3-hidroxi-2,2-dimetilnafto-1,2-pirano (27) [41]. O lapachenol (26) também foi relatado na L. graveolans e L. aff. gracilis [20, 23]. Em L. sidoides também foi isolado uma nova naftoquinona dimérica prenilada, a lippidoquinona (28) [42].

Algumas naftoquinonas são bactericidas e fungicidas enquanto outras apresentam atividade antiprotozoários e antivirais. Entretanto, nenhuma naftoquinona natural é atualmente utilizada com fins terapêuticos [38, 43-44].

\section{Iridóides}

Outro componente presente em espécies de Lippia são os iridóides glicosilados. Eles são produtos do metabolismo secundário de alguns grupos vegetais e desempenham um papel de proteção contra predadores, principalmente herbívoros, por serem venenosos ou amargos $[4,45]$. Nas plantas, os iridóides apresentam uma pronunciada distribuição em angiospermas, notadamente nas Lamiaceae, Verbenaceae, Scrophulariaceae e Plantataginaceae sendo, muitas vezes, utilizados como marcadores taxonômicos a nível de gênero e subgênero [46]. 
Inúmeras publicações demonstram as propriedades biológicas dos iridóides tais como, antiviral, antimicrobiana, antitumoral, hemodinâmica, vasoconstritora, hepatoprotetora e antiinflamatória [47-51].

Rastrelli e colaboradores [52] investigaram as folhas de L. graveolens da Guatemala e isolaram dez iridóides, sendo alguns deles secoiridóides glicosilados. Os menos freqüentes foram secologanina (29), secoxiloganina (30), dimetilsecologanosida (31), ácido logânico (32), loganina (33), ácido 8-epi-loganina (34) e carioptosida (35) e os majoritários foram o ácido carioptosídico (36) e seus derivados 6'-O-p-coumaroil (37) e 6'-O-caffeoil (38).

Os iridóides teviridosídeo (39) e tevesídeo-Na (40) foram isolados das espécies $L$. alba, $L$. javanica, L. turbinata, L. angustifolia, L. arechavaletae, L. ramboi e L. thymoides [53-54].

Nas espécies L. citriodora e L. nodiflora foram identificados genoposídeo (41), presente também na L. alba, ácido genoposídeo-Na (42) e mussaenosídeo (43) [4].

Em 2006, Barbosa e colaboradores [24] isolaram do decocto das folhas de L. alba um novo iridóide chamado shanzshida (44), enquanto que Sena Filho e colaboradores [46] isolaram da mesma planta o teviridosídeo (39), mussaenosídeo (43) e gardosídeo (46). 

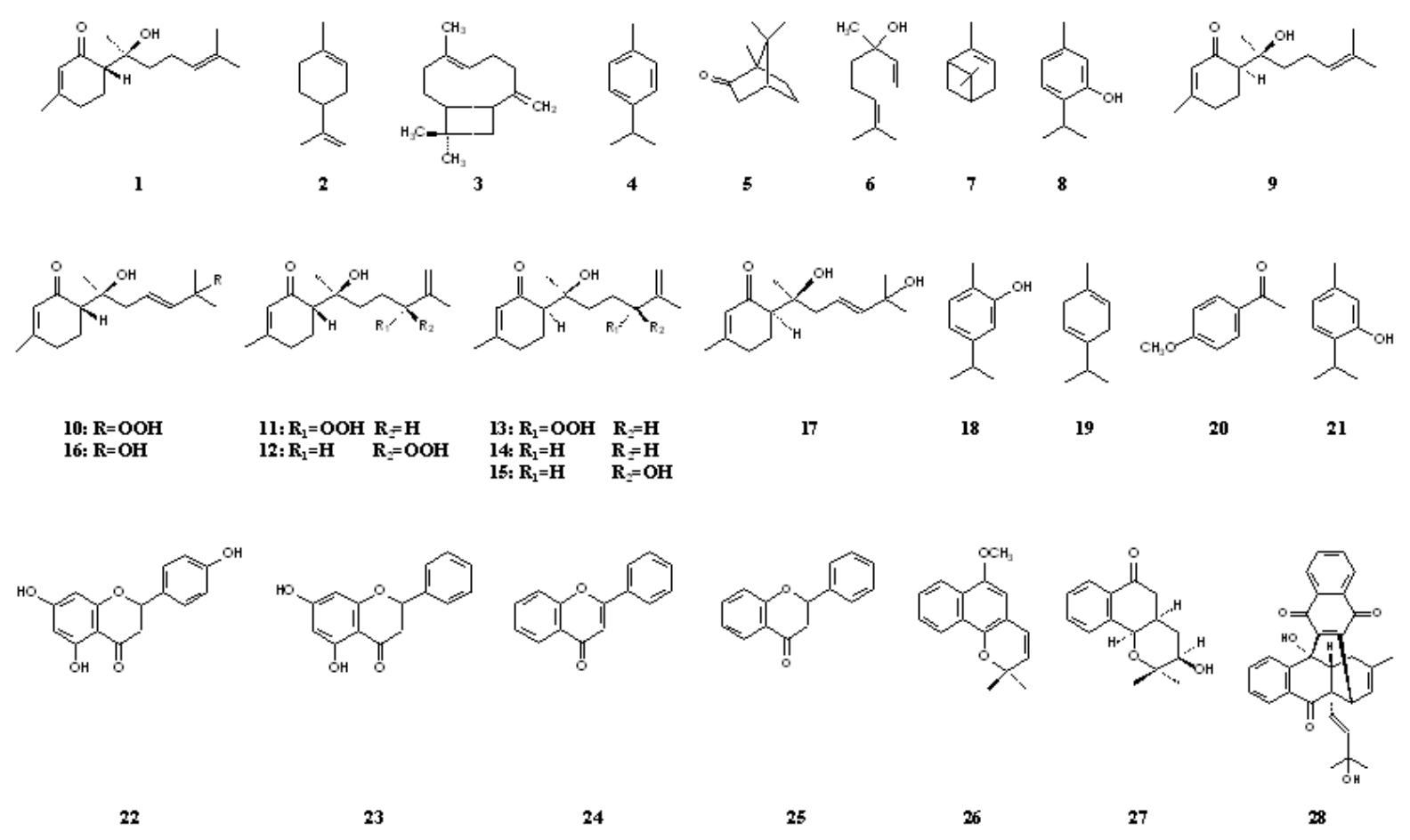

Figura 1: Substâncias isoladas de espécies do gênero Lippia (Verberaceae)
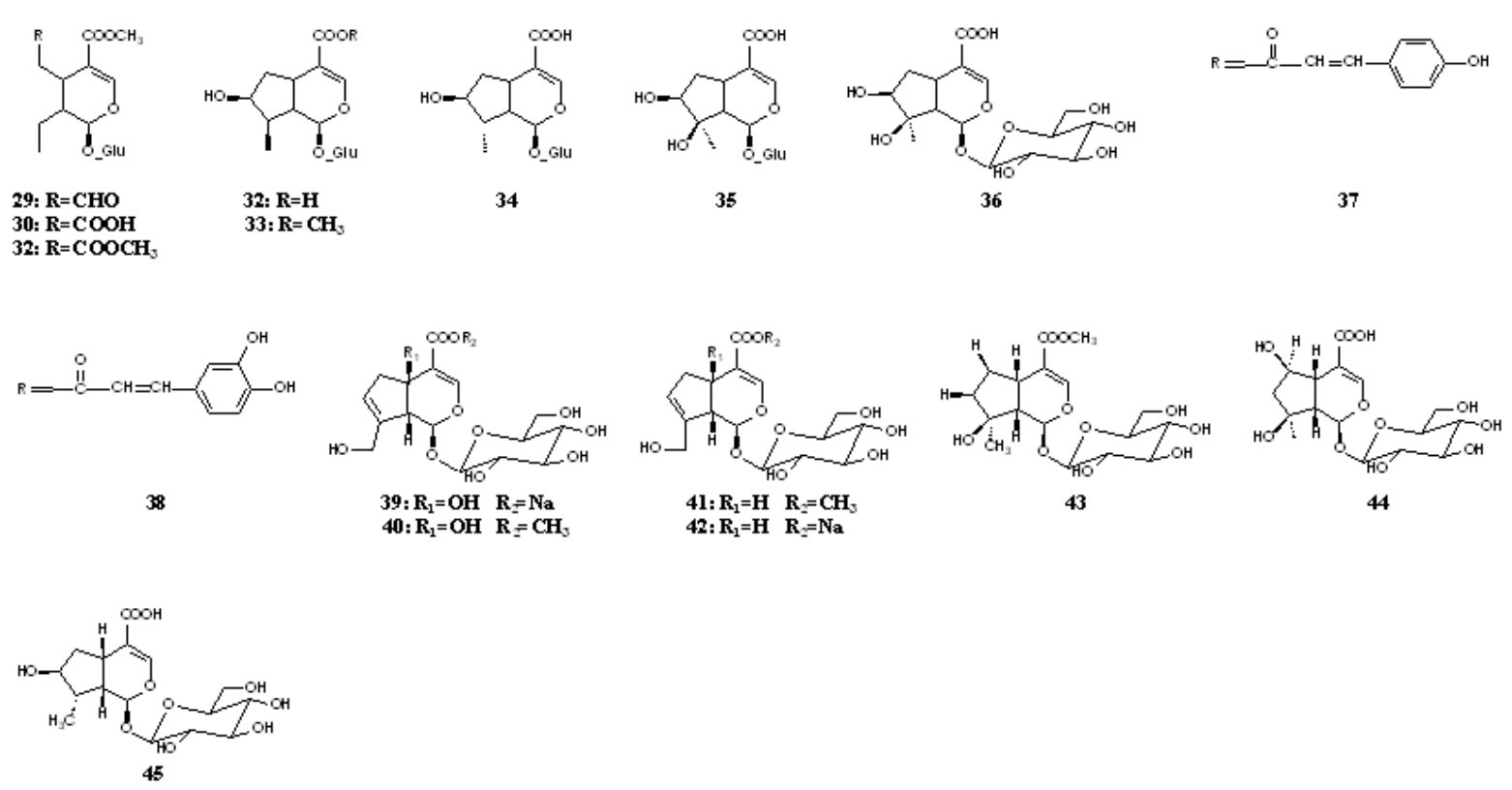

Figura 1: continuação 


\section{Agradecimentos}

À Universidade Federal de Sergipe (UFS) pela bolsa de estudo e ao CNPq.

\section{Referências Bibliográficas}

[1] H. S. Santos, F. M. R. Mesquita, T. L. G. Lemos, F. J. Q. Monte, R. Braz-Filho, Quím Nova 31(3) (2008) 601.

[2] W. S. Judd, Plant Systematics: A phytogenetic approach, Sunderland, UK, Siauer, 2 rd edn., 2002.

[3] F. C. Terblanché, G. Kornelius, J Essent Oil Res. 8 (1996) 471.

[4] M. E. Pascual, K. Slowing, E. Carretero, D. Sánchez Mata, A. Villar, J Ethnopharmacol. 76 (2001) 201.

[5] D. R. Oliveira, G. G. Leitão, H. Bizzo, D. Lopes, D. S. Alviano, C. S. Alviano, S. G. Leitão, Food Chem. 101 (2007) 236.

[6] D. R. Oliveira, G. G. Leitão, S. S. Santos, H. R. Bizzo, D. Lopes, C. S. Alviano, D. S. Alviano, S. G. Leitão, J Ethnopharmacol. 108 (2006) 103.

[7] E. C. Gomes, L. C. Ming, E. A. Moreira, O. G. Miguel, M. D. Miguel, V. A. Kerber, A. Conti, A. Washer Filho, Rev Bras Farm. 60(2) (1993) 103.

[8] S. M. O. Costa, T. L. G. Lemos, F. J. K. Monte, F. J. A. Matos. Simpósio de Plantas Medicinais do Brasil. Águas de Lindóia, São Paulo, 1998.

[9] A. Valentin, Y. Pélissier, F. Benoit, C. Marion, D. Kone, M. Mallie, J. Bastide, J. Bessièri, Phytochemistry 40(5) (1995) 1439.

[10] F. A. Oladimyi, O. O. Orafidiya, T. A. Ogunniyi, F. A. Adewunmi, J Ethnopharmacol. 72 (2000) 305.

[11] C. M. Compadre, E. F. Robbins, A. D. Kinghorn, J Ethnopharmacol. 15(1) (1986) 89.

[12] C. Morton, Atlas of medicinal plants of middle America, Mincis, USA, 1981, chap. 1.

[13] N. Kaneda, I. S. Lee, M. P. Gupta, D. D. Soerjato, A. D. Kinghorn, J Nat Prod. 55(8) (1992) 1136.

[14] R. Guevara, J. Rosales, E. Sanoja, Interciencia 30(10) (2005) 644.

[15] H. Lorenzi, F. J. A. Matos. Plantas medicinais do Brasil: nativas e exóticas cultivadas, Nova Odessa, Instituto Plantarum, 2002. 
[16] T. L. G. Monte, F. J. Q. Matos, F. J. A. Alencar, J. W. Craveiro, A. A. Barbosa, R. C. S. B. Lima, Fitoterapia 63 (1992) 266.

[17] L. M. Girón, V. Freire, A. Alonzo, A. Cárceres, J Ethnopharmacol. 34 (1991) 173.

[18] F. J. A. Matos, Plantas medicinais da medicina popular do Nordeste. Fortaleza, UFC, 1999.

[19] H. Skaltsa, G. Shammas, Planta Med. 54 (1988) 465.

[20] X. A. Domínguez, H. Sánchez, M. Suárez, J. H. Baldas, M. R. González, Planta Med. 55 (1989) 208.

[21] M. De Vicenzi, F. Maialetti, M. R. Dessi, Fitoterapia 66 (1995) 203.

[22] P. Valentão, P. B. Andrade, F. Areias, F. Ferreres, R. M. Seabra, J. Agric. Food Chem. 47 (1999) 4579.

[23] M. C. L. Lima, T. L. G. Lemos, F. J. A. Matos, R. Braz-Filho, O. D. L. Pessoa. $27^{\circ}$ Reunião Anual da Sociedade Brasileira de Química. Salvador, Bahia, 2004.

[24] F. G. Barbosa, M. A. S. Lima, R. Braz-Filho, E. R. Silveira, Biochem Syst Ecol. 34 (2006) 819.

[25] L. Lin, S. Mukhopadhyay, R. J. Robbins, J. M. Harnly, J Food Comp Anal. 20 (2007) 361.

[26] A. L. Bandoni, M. E. Mendiondo, R. V. D. Rondina, J. D. Coussio, J Nat Prod. 35 (1972) 69.

[27] A. Cáceres, L. Fletes, L. Aguilar, O. Ramírez, L. Figueroa, A. M. Taracena, B. J. Samayoa, J Ethnopharmacol. 38 (1993) 31.

[28] A. M. Forestiere, M. T. Monforte, S. Ragusa, A. Trovato, L. Iauk, Phytother Res. 10 (1996) 100 .

[29] K. V. Slowing Barillas. Facultad de Farmácia, Memoria Doctoral, Universidad Complutense de Madrid, 1992.

[30] R. R. Paris, H. Moyses, Matière Médicale, Saint-Germain, Paris, 1971, chap. 3.

[31] A. Hutchings, J. Van Staden, J Ethnopharmacol. 43 (1994) 89.

[32] A. A. Craveiro, J. W. Alencar, F. J. A. Matos, C. H. S. Andrade, M. I. L. Machado, J Nat Prod. 44(5) (1981) 598.

[33] M. Ono, T. Tsuru, H. Abe, M. Eto, M. Okawa, F. Abe, J. Kinjo, T. Ikeda, T. Nohara, J Nat Prod. 69 (2006) 1417.

[34] M. R. A. Santos, R. Innecco, A. A. Soares, Revista Ciência Agronômica 35(2) (2004) 377.

[35] I. A. Neves, J. C. S. Oliveira, C. A. G. Camara, J Essent Oil Res. 20 (2008) 157. 
[36] R. N. Marreto, A. A. S. Araújo, R. S. Nunes, E. C. V. Almeida, A. F. Blank, C. R. S. Matos, E. S. Niculau, P. B. Alves. $31^{\circ}$ Reunião Anual da Sociedade Brasileira de Química. Águas de Lindóia, São Paulo, 2008.

[37] E. Lacoste, J. P. Chaumont, D. Mandin, M. Plumel, F. J. Matos, Ann Pharm Fr. 54 (1996) 228.

[38] A. B. Oliveira, D. S. Raslan, M. C. Miraglia, A. A. L. Mesquita, C. L. Zani, D. T. Ferreira, J. G. S. Maia, Quím Nova. 13(4) (1990) 5.

[39] C. C. Albuquerque, T. R. Camara, R. L. R. Mariano, L. Willadino, C. M. Júnior, C. Ulisses, Braz Arch Biol Techn. 49(4) (2006) 527.

[40] G. D. Carlo, N. Mascolo, A. A. E. Izzo, F. Capasso, Life Sciences, 65 (1999) 337.

[41] L. M. A. Macambira, C. H. S. Andrade, F. J. A. Matos, A. A. Craveiro, J Nat Prod. 49(2) (1986) 310.

[42] S. M. O. Costa, T. L. G. Lemos, O. D. L. Pessoa, J. C. C. Assunção, R. Braz-Filho, Rev Bras Farmacogn. 12 (2002) 66.

[43] R. L. R. P. Jácome, D. S. Raslan, H. Wagner, A. B. Oliveira, Rev Bras Farmacogn. 11(1) (2001) 5 .

[44] R. Y. O. Moreira, M. S. P. Arruda, A. C. Arruda, L. S. Santos, A. H. Muller, G. M. S. P. Guilhon, A. S. Santos, E. Terezo, Rev Bras Farmacogn. 16(3) (2006) 392.

[45] R. Hegnauer, P. Kooiman, Planta Med. 33(1) (1978) 1.

[46] J. G. Sena Filho, J. M. Duringer, D. E. A. Uchoa, H. S. Xavier, J. M. Barbosa-Filho, R. Braz-Filho, Nat Prod Commun. 2 (2006) 715.

[47] S. Takeda, K. Yuasa, T. Endo, M. Aburada, J Pharm Dyn. 3 (1980) 485.

[48] K. Ishiguro, M. Yamaki, S. Takagi, Y. Ikeda, K. Kawakami, K. Ito, T. Nose, J Pharmacobiodyn. 11 (1988) 131.

[49] P. Houghton, H. Hikino, Planta Med. 55 (1989) 123.

[50] M. D. C. Recio, R. M. Giner, S. Manez, J. L. Rios, Planta Med. 60 (1994) 232.

[51] P. Bermejo, M. J. Abad, A. M. Díaz, L. Fernandez, J. De Santos, S. Sanchez, L. Villaescusa, L. Carrasco, A. Irurzun, Planta Med. 68 (2002) 106.

[52] L. Rastrelli, A. Caceres, C. Morales, F. De Simone, R. Aquino, Phytochemistry 49 (1998) 1829.

[53] H. Rimpler, H. Sauerbier, Biochem Syst Ecol. 14 (1986) 307. 
[54] G. L. Von Poster, M. E. Toffoli, M. Sobral, A. T. Henrique, Plant Syst Evol. 205 (1997) 265.

[55] T. Nakamura, E. Okuyama, A. Tsukada, M. Yamazaki, M. Stake, S. Nishibe, T. Deyama, A. Moriya, M. Maruno, H. Nishimura, Chem Pharm Bull. 45(3) (1997) 499.

[56] F. A. Tomás-Barberán, J. B. Harbone, R. Self, Phytochemistry 26 (1987) 2281.

[57] F. J. A. Matos, F. Oliveira, Rev Bras Farm. 79(3/4) (1998) 84.

[58] S. M. O. Costa, T. L. G. Lemos, O. D. L. Pessoa, C. Pessoa, R. C. Montenegro, R. BrazFilho, J Nat Prod. 64 (2001) 792. 